\section{Factors associated with outcome after combined steroid and orbital radiotherapy on Graves' ophthalmopathy}

\author{
Kenji Moriyama,1-3 Kimiko Matsuda, ${ }^{1}$ \\ Hiroshi Arai,2 Takashi Akamizu,2 \\ Tetsuya Tagami,2 Kazuwa Nakao ${ }^{2}$ \\ 1Department of Medicine and Clinical \\ Science, School of Pharmaceutical \\ Sciences, Mukogawa Women's University; \\ 2Department of Medicine and Clinical \\ Science, Kyoto University Graduate \\ School of Medicine; ${ }^{3}$ Department of \\ Nephrology and Blood Purification, \\ Institute of Biomedical Research and \\ Innovation, Kobe Medical Frontier Center, \\ Japan
}

\section{Abstract}

In order to seek possible confounding factors in relation to clinical responsiveness to high-dose intravenous steroid pulse therapy and orbital irradiation followed by oral steroids in the treatment of thyroid-associated ophthalmopathy (TAO), we reviewed the medical records of 53 admitted patients (22 men and 31 women out of a total pool of 167 patients who had diagnosis of TA0). To assess the improvement TAO, we employed proptosis as an objective index and followed up longitudinally. The final ratios of improvement in the left and right eyes respectively were $71.0 \%$ and $64.5 \%$. Significant difference in the therapeutic effect on proptosis was found between male and female at the end of observation. The decrease in proptosis was significantly greater in female patients than in male patients $(\mathrm{P}<0.05)$. Among patients' background characteristics, smoking status and body mass index did not relate to the severity of the eye disease but rather to its improvement (right: $\mathrm{P}=0.18$, left: $\mathrm{P}<0.05)$. These results suggest that the combined steroid and radiotherapy followed by oral steroid therapy should be optimized to obtain higher effectiveness.

\section{Introduction}

Thyroid-associated ophthalmopathy (TA0) is a progressive eye disorder typically associated with Graves' hyperthyroidism. Although TAO also occurs occasionally in patients without hyperthyroidism, these patients often show some abnormalities in thyroid function and immunology.1,2 In the past, several studies documented the effectiveness of intravenous glucocorticoid (IVGC) pulse therapy.3.,4 The rationale for the use of pulse therapy is the observation that TA0, unlike other chronic autoimmune diseases, is most often characterized by a single flare of activity of the autoimmune process. As such, a short-term course of high-dose GC may be effective in impeding/retarding the progression and eventually improving the final outcome of the disease. An overview of the published studies showed a higher percentage of favorable results with the intravenous (IV) route than with the oral (OR) route. ${ }^{3}$ In all patients, GC therapy was used in association with orbital radiotherapy. Those effects are, however, often controversial and not satisfactory, especially in the case of diplopia or proptosis. ${ }^{5}$ Our previous results showed that treatment modalities are associated with favorable effects in the majority of patients. ${ }^{6}$

The aim of the present study was to evaluate whether smoking habits and other confounding factors are still associated with clinical severity and/or improvement of TA0, paying special attention to regression length of proptosis.

\section{Materials and Methods}

\section{Patients and measurements}

We reviewed medical records for patients given a diagnosis of TA0. The investigation period was from January, 1995 to January, 2010. Two patients with contraindications to the use of high-dose systemic GC (insulindependent diabetes) were excluded from the study. The study included 53 patients with moderate-to-severe TAO, defined as progressive proptosis by a clinical activity score (CAS) $\geq 3$ with high intensity signal at the extra-ocular muscles accompanied by evidence of $\mathrm{T} 2$ weighted magnetic resonance imaging scans (MRI). Patients with hypothyroidism or persistent hyperthyroidism were corrected within 2-3 weeks by the administration of T4 or antithyroid drugs, respectively. Doses of oral steroids were determined by patients' body weight. Oral steroid was administered at the end of the first IVGC pulse therapy and followed interpulse period. Assessment of the degree of proptosis and general condition of affected eye were evaluated by well-trained ophthalmologists. Exophthalmometry were performed a minimum of four times: i) pretreatment, ii) just after high dose IVGC therapy, iii) at discharge, and iv) at the last day of consultation. Smoking status was determined from a questionnaire at the time of hospitalization (Table 1).
Correspondence: Kenji Moriyama, Department of Medicine and Clinical Science, School of Pharmaceutical Sciences, Mukogawa Women's University, 11-68 Koshien-kyubancho Nishinomiya, 663-8179, Japan.

Tel. +81.0798 .459930 - Fax: +81.0798 .459930 .

E-mail: kemori@mukogawa-u.ac.jp

Key words: intravenous steroid pulse therapy, thyroid, ophthalmometry.

Acknowledgements: the authors are grateful to Dr. S. Kashii for kindly performing ophthalmological examinations of the present cases and to Dr. Y. Nagata and Dr S. Itasaka for performing radiotherapy on the patients.

Funding: this work was supported by Ministry of Science and technology foundation (No. 22590 614 to K. M.) to KM.

Contributions: KM and KM contributed to make the data base and analysis. HA, TA, TT and KN contributed to give substantial comments on the manuscript. Initial preparation of the manuscript was carried out by KM with revisions and final approval from all other authors.

Conflict of interests: the authors report no potential conflict of interests.

Received for publication: 15 January 2013.

Revision received: 13 March 2013.

Accepted for publication: 29 March 2013.

This work is licensed under a Creative Commons Attribution NonCommercial 3.0 License (CC BYNC 3.0)

(C) Copyright K. Moriyama et al., 2013

Licensee PAGEPress, Italy

Endocrinology Studies 2013; 3:e6

doi:10.4081/es.2013.e6

\section{Standard regimens of intravenous}

glucocorticoid and oral glucocorticoid

Methylpredonisolone (MP) as IVGC $1000 \mathrm{mg}$ was used for high-dose intravenous pulse therapies. A course of steroid pulse therapy is comprised of 3 consecutive days of administration (1000 mg/day) at 4-day intervals. Total number of steroid pulse therapies of a patient was three. Predonisolone (PSL) as ORGC was also administered in the final course of the pulse therapy. Initial dose of PSL was decided by the body weight of the patient at a ratio of 1 $\mathrm{mg} / \mathrm{kg} /$ day (maximal dose was up to $60 \mathrm{mg}$ ). Doses were then gradually tapered by 5 mg/week and terminated within 3-12 months.

\section{Radiation therapy}

After the pulse therapy, orbital radiotherapy was administered within 2 weeks after completion of pulse therapies. A computer-assisted tomography of the orbit was performed in all 
patients before treatment to define the radiation field. All patients were treated bilaterally. Irradiation of the orbit of the side(s), in which the swelling of extraocular muscle(s) was found by MR imaging, was performed with an $\mathrm{X}$-ray beam with an energy of $10 \mathrm{MeV}$ generated by a linear accelerator. ${ }^{6}$ Ten daily doses of 200 cGy to each eye were given over 2 weeks for a cumulative dose of $20 \mathrm{~Gy}$.

\section{Ocular evaluation}

All patients were examined before GC treatments, before beginning of irradiation treatment, upon discharge, and at 3-6 month intervals thereafter. Exophthalmometry were performed for each patient at each visit. Progression or improvement of ophthalmopathy was defined by changes in the length of proptosis (Inami Hertel exophthalmometer). The mean time elapsed between the start of the therapy and the final assessment of TAO as clinical responses after the therapy was $286 \pm 188$ days (range $80-1223$ days).

\section{Statistical analysis}

Length of proptosis was chosen as a severity objective index in determining primary outcome measurements, as this manifestation most closely delineates disease. The mean duration of follow up is (mean \pm SD) $286 \pm 188$ days (range 80-1223 days). Data were analyzed for statistical significance by analysis of variance in combination with Student's unpaired and paired t-test. Changes from baseline in successive three endpoints were analyzed by one-way analysis of variance (ANOVA). We employed a cox proportional hazards model to estimate the appearance time of combinatorial treatment effects. As a censored variable, more than $0.5 \mathrm{~mm}$ decrease in length of proptosis in comparison with baseline was employed. The analysis used SPSS version 17.0 (SPSS Inc., an IBM Company, Illinois). $\mathrm{P}<0.05$ was considered to be statistically significant.

\section{Results}

\section{Clinical baseline of the patients}

When we conducted exophthalmometry during the hospitalizations, we did not recognize the statistical significant difference for the severity of proptosis between male and female (Table 1). Differences in age and BMI between males and females were statistically significant. Endocinological examinations of the patients at baseline were also shown in Table 1 . TRAbs between males and females were statistically different. We did find there was no relationship between proptosis and endocrinological examinations of the thyroid so far (data not shown). We also found no association between
Table 1. Clinical baseline of the patients.

\begin{tabular}{|c|c|c|c|c|}
\hline & $\begin{array}{c}\text { Total } \\
(\mathrm{n}=53)\end{array}$ & $\begin{array}{c}\text { Male } \\
(n=21)\end{array}$ & $\begin{array}{l}\text { Female } \\
(n=32)\end{array}$ & $\begin{array}{c}\text { Unpaired student } \\
\text { t-test } \\
\text { (P value) }\end{array}$ \\
\hline Age at hospitalization & $50.5 \pm 11.6$ & $52.0 \pm 11.4$ & $49.4 \pm 11.6$ & 0.05 \\
\hline BMI at pre-treatment $(\mathrm{kg})$ & $22.3 \pm 3.2$ & $23.6 \pm 4.7$ & $21.5 \pm 3.5$ & 0.05 \\
\hline $\begin{array}{l}\text { Smoking status } \\
\text { Smoker } \\
\text { Non-smoker } \\
\text { Ex-smoker }\end{array}$ & $\begin{array}{c}21 \\
29 \\
3\end{array}$ & $\begin{array}{c}11 \\
9 \\
1\end{array}$ & $\begin{array}{c}10 \\
20 \\
2\end{array}$ & - \\
\hline Proptosis right $(<17 \mathrm{~mm})$ & $19.7 \pm 4.0$ & $19.2 \pm 4.3$ & $20.1 \pm 3.8$ & 0.43 \\
\hline Proptosis left $(<17 \mathrm{~mm})$ & $19.7 \pm 3.6$ & $19.1 \pm 2.7$ & $20.3 \pm 4.1$ & 0.26 \\
\hline $\begin{array}{l}\text { Endocrinological examinations } \\
\text { TSH }(0.500-5.00 \mu \mathrm{IU} / \mathrm{mL}) \\
\text { Free T4 }(0.90-1.70 \mathrm{ng} / \mathrm{dL}) \\
\text { Free T3 }(2.30-4.30 \mathrm{ng} / \mathrm{dL}) \\
\text { TRAb }(\leq 15 \%) \\
\text { TRAb }(1.0<\mathrm{IU}) \\
\text { TSAb }(\leq 180 \%)\end{array}$ & $\begin{array}{c}2.74 \pm 5.35 \\
1.63 \pm 1.13 \\
3.68 \pm 1.95 \\
27.8 \pm 26.0 \\
13.2 \pm 13.9 \\
1007.4 \pm 1064.7\end{array}$ & $\begin{array}{c}2.2 \pm 2.71 \\
1.76 \pm 1.21 \\
3.89 \pm 1.69 \\
15.9 \pm 15.1 \\
6.4 \pm 3.3 \\
969.4 \pm 1278.9\end{array}$ & $\begin{array}{c}2.92 \pm 6.38 \\
1.54 \pm 1.08 \\
3.59 \pm 2.13 \\
35.4 \pm 29.0 \\
17.0 \pm 16.4 \\
1037 \pm 891.4\end{array}$ & $\begin{array}{l}0.78 \\
0.50 \\
0.55 \\
0.05 \\
0.01 \\
0.85\end{array}$ \\
\hline
\end{tabular}

BMI; body mass index. Results are expressed \pm standard deviation. Parameters in parenthesis indicate the normal ranges.
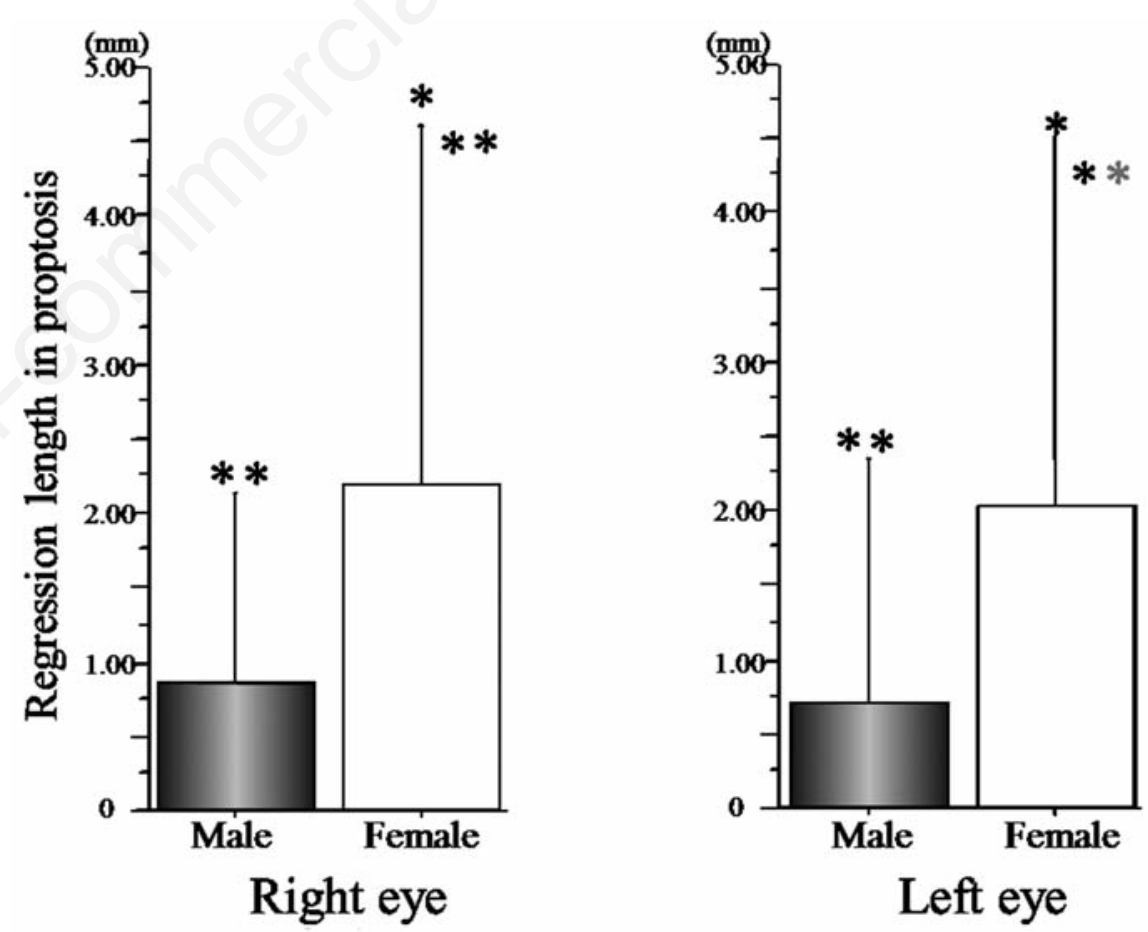

Figure 1. Relationship between sex and clinical improvement in proptosis. Mean regression lengths in proptosis after the final course of steroid pulse therapies were calculated and compared between male and female using the Student $t$ test. Although proptosis at pretreatment were not statistically significant by sex (right: $P=0.43$, left: $P<0.26$ ), the overall responses for combination therapies were more effective for female than male patients in particular. ${ }^{*} \mathrm{P}<\mathbf{0 . 0 5}$ comparison between male and female. ${ }^{* *} \mathrm{P}<\mathbf{0 . 0 1}$ comparison between after and before treatment. 
smoking status and biochemical and endocrinological examinations (data not shown).

\section{Proportion of subjects with clinical response following steroid pulse therapy}

It took 379 days to response to steroid and orbital irradiation therapy in a half of the patients as calculated with the Kaplan-Meier method (Figure 1). Overall, those routine treatments produced favorable effects in most patients by the time of discharge, but responders of the treatment comprised less than $69.4 \%$ (right: $71.0 \%$, left: $64.5 \%$ ). The remainder were not clinically responsive in evaluation of proptosis by the end of observation.

\section{The overall response for combined}

\section{therapies}

Relationship between sex and clinical improvement in proptosis is shown in Figure 1.

Mean regression lengths in proptosis at the time of discharge therapies and the end of observation were calculated and compared between male patients and female patients. Although proptosis at pretreatment were not statistically significant by sex (right: $\mathrm{P}=0.43$, left: $\mathrm{P}=0.26$ ), significant reduction occurred in both sexes at the end of observation, with no difference between the right and left eyes. However, the grade of clinical improvement in proptosis was significantly greater in female patients than in male patients.

Mean lengths in proptosis were calculated and compared among tertiles of BMI (13.6 \pm 28.8$)$. Results show the following correlation, the lighter the tertile of BMI, the better regression in proptosis. We found those mild associations continuing through the observation period (Figure 2).

As for relationship between smoking habit and clinical aspects in proptosis, mean lengths at pretreatment based on smoking status was not statistically significant (right: $\mathrm{P}=0.92$, left: $\mathrm{P}=0.14$ ). By smoking status, mean regressing lengths in proptosis were statistically significant at the end of observation (mean values \pm SD were as follows: the right eye: non-smoker; $2.5 \pm 2.5$, smoker; $1.2 \pm 3.0, \mathrm{P}<0.05$, the left eye: non-smoker; $2.1 \pm 3.4$, smoker; $1.3 \pm 2.6$, $\mathrm{P}<0.05)$. The outcome of exophthalmometry at final observation were as follows: non-smokers' mean values \pm SD of the right eye: $16.7 \pm 4.0 \mathrm{~mm}$, left: $16.2 \pm 4.1 \mathrm{~mm}$; smokers' right: $18.3 \pm 4.2$, left: $19.4 \pm 3.6 \mathrm{~mm}$ respectively (Figure 3). Among patients' background variables, smoking status did not relate to the severity of exophthalmos. On the contrary, smoking actually showed a positive correlation to its improvement.

\section{Discussion}

Thyroid-associated ophthalmopathy, the most frequent extrathyroidal manifestation of Graves' disease, is a disorder of autoimmune origin, the pathogenic mechanisms of which are still incompletely understood. Although TAO is severe in only 3-5\% of affected individuals, quality of life is severely impaired even in patients with mild TA0. ${ }^{7-10}$ Management of TAO can be either medical or surgical. Medical management relies on the use of high-dose systemic GC or orbital radiotherapy, either alone or in combination. ${ }^{4,11,12}$ However, control of active TAO remains a difficult task, and many patients are not satisfied with the effects of treatments, especially with the appearance of their eyes. ${ }^{10} \mathrm{GC}$ therapy in association with orbital radiotherapy is one of the established and most commonly used treatments for TAO, and seems to be effective., ${ }^{4,13,14}$ Indeed, a favorable response has been reported.,13-16 They are used in view of their anti-inflammatory and immunosuppressive actions..$^{17}$ Most commonly, GC has been used through the oral or local (retrobulbar or subconjunctival) routes, ${ }^{16}$ but locally administered GC is not as effective as systemic GC treatment. ${ }^{17}$ On the other hand, oral GC therapy requires the use of high doses
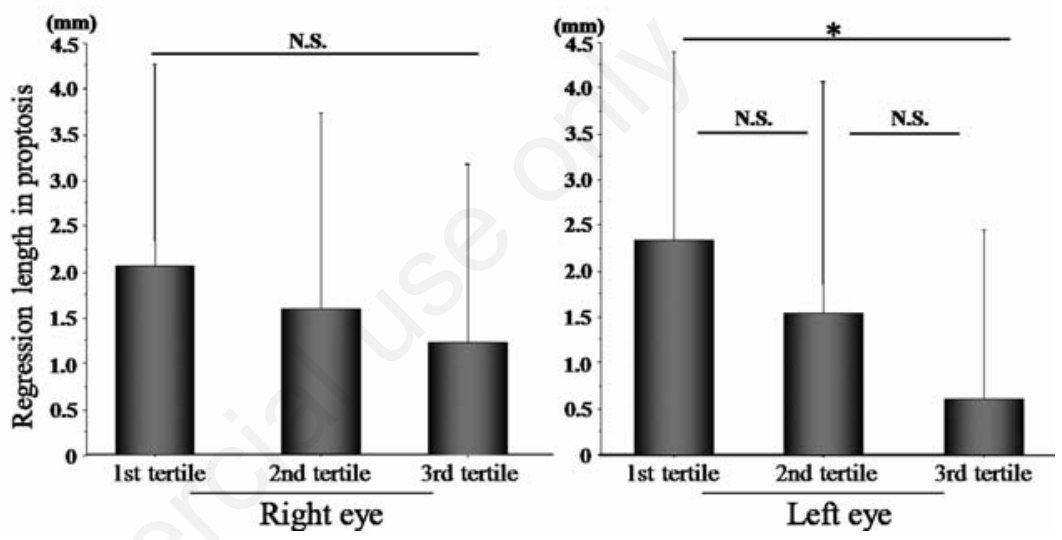

Figure 2. Relationship between body mass index (BMI) and clinical improvement in proptosis. To compare relationship between BMI and clinical improvement in proptosis, all patients were categorized into 3 subgroups according to BMI. The lighter tertile of BMI was, the better regression in proptosis was. Parametric comparisons among 3 groups were performed with ANOVA. Significance of individual difference was evaluated by using the Scheffes' test, if ANOVA was significant. Results were expressed as mean \pm standard deviation. ${ }^{*} P<0.05$ comparison between 1 st and 3 rd tertiles.
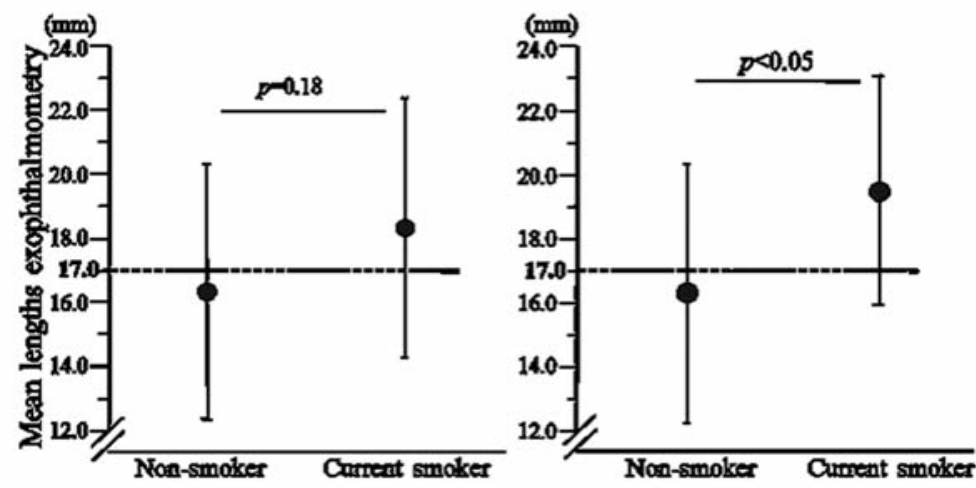

Figure 3. Relationship between smoking habit and exophthalmometry as final outcomes. Mean lengths in proptosis after 6-24 months later from the day of discharged were calculated and compared between patients with and without smoking habits using the Student $t$ test. Results were expressed as mean $\pm S D$, (right: $P=0.18$, left: $P<0.05)$. The broken line indicates the boundary of normal range of proptosis in NOSPCES. 
of prednisolone (60-100 mg/day, or equivalent) for prolonged periods of time (usually several months). ${ }^{8}$ The use of radiotherapy in conjunction with GC treatment has been shown to prevent the rebound of the inflammatory manifestations that are often seen after GC treatment alone. ${ }^{\text {? }}$

The natural history of TAO is incompletely defined. The onset of TAO is in most cases concomitant with the onset of hyperthyroidism, but TAO may precede or follow hyperthyroidism. ${ }^{18}$ As the factors concerning the onset, severity, and drug responsiveness of TAO are yet uncertain and the role that hyperthyroidism management may play on TA0's emergence and development are also unclear, TAO management remains a key research topic. ${ }^{19}$ In this review, we found therapeutic indication for TAO in 53 out of 167 patients. The remainders who showed no medical signs judging from CAS and MRI had missed their particular window of responsiveness to treatment mainly due to a gap between pathological activities and clinical symptoms.

In this report, we had employed the proptosis as an objective marker to evaluate disease severity and drug responsiveness of TAO statistically. The significant reduction in proptosis occurred in both sexes. However, the grade of clinical improvement in proptosis was significantly greater in female patients than in male patients. There was not the sex-related difference at all at the commencement of treatments, except for TRAbs and TSAb (Table 1). Although the complete explanation is difficult, the higher titer of anti-TSH receptor antibodies in female may be related to the responsiveness to high dose steroids.

TAO is a local orbital inflammatory disease in which de novo adipogenesis has been identified as a fundamental pathogenic mechanism. ${ }^{20} 11$-Hydroxysteroid dehydrogenase 1 increases cortisol bioavailability and is playing pivotal role in mediating glucocorticoid responses in adipose tissue and inflammation. ${ }^{20}$ Those are common etiopathogenesis to both TAO and metabolic syndrome. ${ }^{21}$ So we chose BMI to test the significance as a variable. To compare the relationship between BMI and clinical improvement in proptosis, patients were divided evenly into three subgroups according to their BMI. We found a mild correlation in the comparison among them, although there were no differences in proptosis at the commencement of treatments. The BMI and clinical improvement association remained constant throughout the observation period, as the subject's BMI was tightly controlled. Besides sex, BMI is an additional possible confounding factor. We could not produce definitive reasons for the mild correlation between them so far. Thiazolidines are recommended to be withheld for patients with diabetes and active TAO due to increase in size and number of adipocytes. Systemic adipocytes and/or adipocytokines might be in relation to amelioration of TA0.22

An association has been reported between TAO and smoking. 8,23 Previous reports indicate that smoking greatly increased the risk for TA0, but patients with Graves' hyperthyroidism alone were also more often smokers than control subjects. ${ }^{24,25}$ Furthermore, smoking is also associated with a higher degree of disease severity. ${ }^{25}$ We performed several comparisons to ascertain any possible links between smoking and TAO and to assess the conditions under which this association would persist as a confounding factor. In our data, smoking was not associated with the severity of proptosis, though the rate of non-Japanese patients with a smoking history may be higher than that of Japanese patients, especially of female patients. We could not find any significant differences in the outcomes of exophthalmometry in both smokers and non-smokers at the beginning of therapies. Indeed, the mean initial proptosis was significantly reduced at the end of observation after the therapies in both groups, but the mean degree of improvement in TA0 was much higher in non-smokers than in smoker patients. These results suggest that smoking habit in TAO still belongs to confounding factors. 8,25 Thus, smoking appears to be one of the controllable factors deciding curative effects of TAO in genetically predisposed individuals.

Although the previous report showed that GC therapy exerted the greatest effectiveness on optic nerve involvement and soft tissue changes, proptosis was less responsive. ${ }^{26}$ Ohtsuka and their colleagues carried out a prospective study to evaluate the effect of high-dose IVGC pulse therapy (1000 mg/day x 3 consecutive days by 3 weeks) followed by 3 month oral steroid therapy for 41 Japanese patients with Graves' ophthalmopathy. ${ }^{27}$ The report said that proptosis was not significantly reduced by the steroid pulse therapy between pre and post (months 1-6).27 Matejka et al. investigated the efficacy of IVGC pulse therapy (a $12.5 \mathrm{mg} / \mathrm{kg}$ dose of MP once every month) on TAO in eight patients. ${ }^{28}$ They followed up for at least 6 months by clinical ophthalmological examination. They concluded that MP pulse therapy had less effect on proptosis, though affected eyes were improved from $22.94 \pm 2.32$ $\mathrm{mm}$ to $21.56 \pm 2.22 \mathrm{~mm} \quad(\mathrm{P}<0.05) \quad(2-77$ months). ${ }^{29}$ Improvement in proptosis was found in at least $64.5 \%$ of patients in either the right and/or left eyes (right: 71.0\%, left: $64.5 \%)$. The balance (35.5\%) were not responsive in the evaluation of regression length of proptosis by the current regimens. In an analysis of published studies-all nonrandomized-the mean percentage of responders was $77 \%$ in patients treated IVGC and $63 \%$ in patients treated ORGC..$^{29}$ Evaluating proptosis as a ther- apeutic index, we also got the same level of the effect with current protocol. In the present investigation, we signified proptosis as a clinical manifestation and therapeutic index by which to evaluate combined therapies comprised of IVGC associated with ORGC treatment during subsequent period and orbital radiotherapy. Calculating with the KaplanMeier method, the overall responsiveness to the combined therapy in a half of the patients was over a year. Our results indicate that combined treatment modalities are effective and may take over 12 months to evident in the majority of patients as final results. However, there were various individual differences of the time and confounding factors required for therapeutic effects to manifest.

\section{Conclusions}

Though therapies for TAO range from medical therapies for active stage to surgical treatment for burnt-out, both high-dose steroids and orbital radiotherapy may still be the mainstay of medical therapy. So it is mandatory to optimize whether confounding factors is present, as this requires appropriate treatment.

\section{References}

1. Weetman AP. Thyroid-associated eye disease: pathophysiology. Lancet 1991;338:258.

2. Wall JR, Bernard N, Boucher A, et al. Pathogenesis of thyroid-associated ophthalmopathy: an autoimmune disorder of the eye muscle associated with Graves' hyperthyroidism and Hashimoto's thyroiditis. Clin Immunol Immunopathol 1993;68:1-8.

3. Kauppinen-Mäkelin R, Karma A, Leinonen E, et al. High dose intravenous methylprednisolone pulse therapy versus oral prednisone for thyroid-associated ophthalmopathy. Acta Ophthalmol Scand 2002;80: 316-21.

4. Stiebel-Kalish H, Robenshtok E, Hasanreisoglu M, et al. Treatment modalities for Graves' ophthalmopathy: systematic review and meta-analysis. J Clin Endocrinol Metab 2009;94:2708-16.

5. Wiersinga WM. Immunosuppressive treatment of Graves ophthalmopathy. Thyroid 1992;1:229-33.

6. Tagami T, Tanaka K, Sugawa H, et al. Highdose intravenous steroid pulse therapy in thyroid-associated ophthalmopathy. Endocr J 1996;43:689-99.

7. Wiersinga WM, Bartalena L. Epidemiology and prevention of Graves' ophthalmopathy. 
Thyroid 2002;12:855-60.

8. Bartalena L, Baldeschi L, Dickinson A, et al. European Group on Graves' Orbitopathy (EUG0G0): Consensus statement of the European Group on Graves' orbitopathy (EUGOG0) on management of G0. Eur J Endocrinol 2008;158:273-85.

9. Bartalena L, Marcocci C, Tanda ML, et al. An update on medical management of Graves' ophthalmopathy. J Endocrinol Invest 2005;28:469-78.

10. Wiersinga WM. Quality of life in Graves' ophthalmopathy. Best Pract Res Clin Endocrinol Metab 2012;26:359-70.

11. Ohtsuka K, Sato A, Kawaguchi S, et al. Effect of steroid pulse therapy with and without orbital radiotherapy on Graves' ophthalmopathy. Am J Ophthalmol 2003;135:285-90.

12. Koshiyama H, Koh T, Fujiwara K, et al. Therapy of Graves' ophthalmopathy with intravenous high-dose steroid followed by orbital irradiation. Thyroid 1994;4:409-13.

13. Konishi J, Iida Y, Kasagi K, et al. Clinical evaluation of radiotherapy for Graves' ophthalmopathy. Endocrinol Jpn 1986;33:63744.

14. Marcocci C, Marinò M. Treatment of mild, moderate-to-severe and very severe Graves' orbitopathy. Best Pract Res Clin Endocrinol Metab 2012;26:325-37.

15. Donaldson SS, Bagshaw MA, Kriss JP. Supervoltage orbital radiotherapy for Graves، ophthalmopathy. J Clin Endocrinol Metab 1973;37:276-85.

16. Marcocci C, Bartalena L, Panicucci M, et al. Orbital cobalt irradiation combined with retrobulbar or systemic corticosteroids for Graves' phthalmopathy: a comparative study. Clin Endocrinol 1987;27:3342.

17. Burrow GN, Mitchell MS, Howard RO. Immunosuppressive therapy for the eye changes of Graves' disease. J Clin Endocrinol Metab 1970;31:307-11.

18. Salvi M, Zhang Z-G, Haegert D, et al. Patients with endocrine ophthalmopathy not associated with overt thyroid disease have multiple thyroid immunological abnormalities. J Clin Endocrinol Metab 1990; 70:89-94.

19. Gopinath B, Musselman R, Adams CL, et al. Study of serum antibodies against three eye muscle antigens and the connective tissue antigen collagen XIII in patients with Graves' disease with and without ophthalmopathy: correlation with clinical features. Thyroid 2006;16: 967-74.

20. Tomlinson JW, Durrani OM, Bujalska IJ, et al. The role of 11beta-hydroxysteroid dehydrogenase 1 in adipogenesis in thyroidassociated ophthalmopathy. Clin Endocrinol Metab 2010; 95:398-406.

21. Putignano P, Pecori Giraldi F, Cavagnini F. Tissue-specific dysregulation of 11betahydroxysteroid dehydrogenase type 1 and pathogenesis of the metabolic syndrome. $\mathrm{J}$ Endocrinol Invest 2004; 27:969-74.

22. Starkey K, Heufelder A, Baker G, et al. Peroxisome proliferator-activated receptor-gamma in thyroid eye disease: contraindication for thiazolidinedione use? J
Clin Endocrinol Metab 2003;88:55-9.

23. Stan MN, Bahn RS. Risk factors for development or deterioration of Graves' ophthalmopathy. Thyroid 2010;20:777-83.

24. Winsa B, Mandahl A, Karlsson FA. Graves' disease, endocrine ophthalmopathy and smoking. Acta Endocrinol (Copenh) 1993;128:156-60.

25. Prummel MF, Strieder T, Wiersinga WM. The environment and autoimmune thyroid diseases. Eur J Endocrinol 2004;150:60518.

26. Marcocci C, Bartalena L, Tanda ML, et al. Comparison of the effectiveness and tolerability of intravenous or oral glucocorticoids associated with orbital radiotherapy in the management of severe graves' ophthalmopathy: results of a prospective, single-blind, randomized study. J Clin Endocrinol Metab 2001;86:3562-67.

27. Ohtsuka K, Sato A, Kawaguchi S, et al. Effect of high-dose intravenous steroid pulse therapy followed by 3 -month oral steroid therapy for Graves' ophthalmopathy. Jpn J Ophthalmol 2002;46:563-7.

28. Matejka G, Vergès B, Vaillant $G$, et al. Intravenous methylprednisolone pulse therapy in the treatment of Graves' ophthalmopathy. Horm Metab Res 1998;30:938.

29. Bartalena L, Pinchera A, Marcocci C. Management of Graves' ophthalmopathy: reality and perspectives. Endocr Rev 2000; 21:168-99. 